\title{
Hosting and Sharing Your Own Remote Experiments with RALfie - an Open Ended Experiment Design Experience
}

\author{
http://dx.doi.org/10.3991/ijoe.v12i04.5101 \\ Alexander Kist, Ananda Maiti, Andrew Maxwell, Lindy Orwin, \\ Wu Ting, Peter Albion and Ryan Burtenshaw \\ University of Southern Queensland, Australia
}

\begin{abstract}
Remote Access Laboratories (RAL) are online platforms for performing experiments from remote locations usually following a centralised service oriented paradigm. This short article presents RALfie - Remote Access Laboratories for Fun, Innovation and Education using a peer-to-peer RAL architecture where some participants or makers can create, host and share experiments for other users. The system is built upon a VPN service that establishes end-to-end connections between learner and makers' experiential rigs. A graphical programming platform SNAP is the basis of programming and designing the interface with the experimental rig. The experiments are then shard using a quest-based learning strategy that presents the experiments as a set of hierarchical groups of activities or quests. This distributed design of RAL allows more hands-on experience to build experimentals setup and provides opportunities to collaborate with fellow peers. The environment is suitable foe STEM Education. This approach requires the makers among the users to create and host the experiments, which demand certain responsibilities and offers new learning opportunities.
\end{abstract}

Index Termss-remote laboratories;STEM education; makers.

\section{INTRODUCTION}

Laboratory activities play an important role in science and engineering education. There have been many projects to make laboratory activities available online via the Internet. Some of the major initiatives include iLab, Labshare and VISIR [1]. Most activities are developed and hosted by Universities and used for undergraduate teaching. More recently remote laboratories have also been proposed in the context of school education [2,3]. Traditionally, remote laboratories are hosted in centralized locations and access to the experiments largely follows a client-server paradigm. Experiments are built by experts and used by students. However, this does not reflect the way experimental learning is traditionally used in schools. Here setting up the experiment and building the apparatus or rig from an important part of the learning activity.

An alternative to the traditional, centralized approach are peer-to-peer remote access laboratories were the experiments are designed, build and hosted in a distributed manner by students. In such an environment students are provided with the necessary tools to conceive, design and build experiments. Those are shared with others who run the activities remotely. Both the maker-experience as well as the user-experience is important learning activities. To make a system like this possible a number of challenges need to be addressed. The approach of RALfie and some of the key solutions are briefly discussed in the next Sections.

\section{THE PROJECT}

Remote Access Laboratories for fun, innovation and education (RALfie) is a collaborative research project between academics from engineering and education with the aim to promote STEM (Science, Technology, Engineering and Mathematics) subjects among young learners. RALfie employs at the users' level a Peer-to-Peer approach where the users build remotely accessible experiments to be shared by others. This system makes direct collaboration possible between students which can be supported by their teachers. It also allows them to develop and design solutions to problems thus gaining hands-on experience. The activities are integrated into and shared through a online quest-based engagement framework. The process of creating and hosting of experiments includes the following activities: assembling a rig, programming experiment control, implementing the user interface and connecting the experiment to the Internet [4].

The experiments created with this approach are generally simpler and often used improvised components in their rigs compared to the traditional remote laboratories. This approach uses a low cost model of building an experiment with commercially available consumer tools e.g. microcontroller units, sensors and actuators. Once the experiments are ready, they are published into the quest-based learning system which allows the other users to search as per their needs and access the appropriate experiments.

To realize the aims of the project a number of issues had to be addressed. This included

1. enabling a managed end-to-end connectivity between experiment and learner devices,

2. providing an engagement and collaboration model,

3. providing a way how the experiments are controlled and

4. addressing how online user interfaces and associated learning materials are designed.

\section{RAlfie System}

The first objective was to design a platform to create experiment rigs of multiple types and configurations while using the same set of control commands. Also, the experiments had to be able to connect to the Internet and y the learner site through the P2P RALMS. 


\section{A. Hosting Experiments - Making and Controll}

Micro-controller units are used to control experiments in RALfie. These controllers have multiple ports connected to different sensor and actuators. They provide a generic platform for building a variety of experiments. The different controllers that are used include LEGO Mindstorms EV3, Arduino, BeagleBone Black and Raspberry Pi. All have different characteristics and varying advantages regarding communication and control [5].

Part of the RALfie experience is to make an experiment. As hands-on-experience is one of the kay goals of RALfie, the entire system is designed to cope with a wide range of devices and their configurations. The makers can build any kind of rig they want to and plug them into the RALfie system. Fig 1 shows the maker end architecture involving the MCU, peripheral devices and connection mechanism to the internet through a RALfieBox.

Each MCU is composed of a processor, memory and network interface. The MCU and the IP Camera connects to the RALfieBox. The MCUs are programmed to receive and parse instructions from the users and respond to them. The makers may come up with different kinds of designs for the same activity.

\section{B. End-to-End Connectivity}

To safely access experiments remotely, access control, authentication and mediation are necessary. This is particularly important in the context of working with children. In our system this is addressed by providing each experiment site with an access gateway ("RALfie Box") that joins a VPN when connected to the Internet. The local experiments and cameras are connected to the RALfie Box [3] and experiment access is provided via a central server that forwards requests via the VPN to the experiment sites.

\section{DELIVERY TO USERS}

Once the experiment setup has been designed, it has to be programmed and presented to the users in a manner that is both educationally sound and appealing for users.

\section{A. Experiment logic and user interface programming}

Two aspects require programming skills of the user -

- writing the control logic for the experiments and

- creating graphical (online) user interfaces.

While some users may have been exposed to programming activities, this is not a universal skill. However, there are a number of programming languages and environments used to teach young learner to programming; for example Scratch [6] by the Lifelong Kindergarten Group at the MIT Media Lab and SNAP an extended reimplementation of Scratch at Berkeley. Both are visual, dragand-drop programming languages running locally in a browser. In the RALfie project we are using SNAP with customisations to interface with the RALfie system (see Fig 2).

The RALfie environment provides special blocks related to the MCUs. The makers can incorporate these blocks in their program to manipulate the operations of the rigs. The control components are simple images as created by the makers and put on the UI.

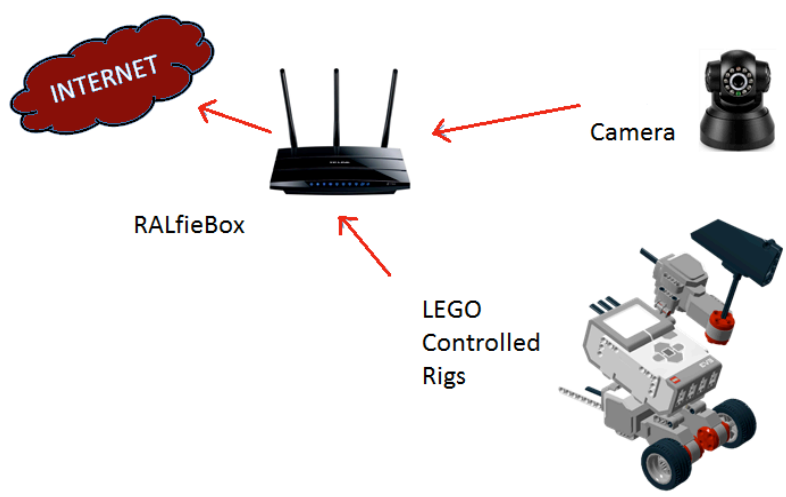

Figure 1. The RALfie maker end

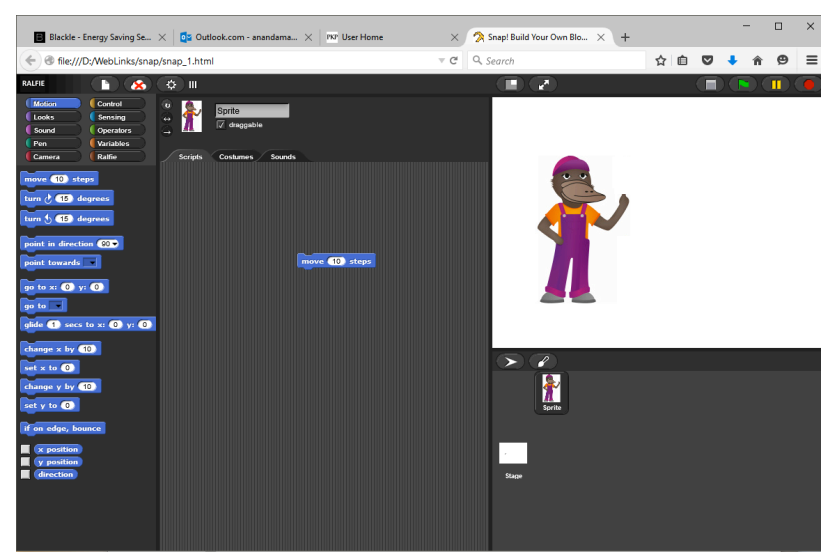

Figure 2. The SNAP programming Interface

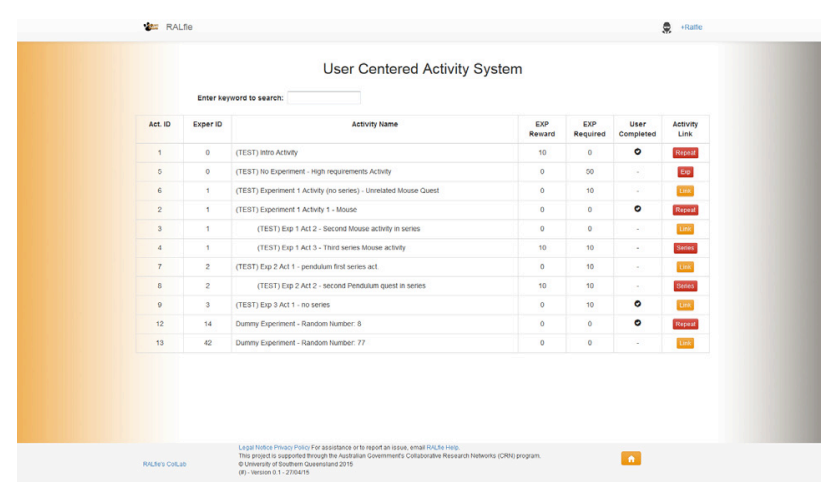

Figure 3. The game webpage of the RALfie system

\section{B. Game-Based Learning Environment}

All experimental rigs in the system are part of a quest or activities that involves using the rigs to produce outputs. Upon completing of quests, the user gains experience points and other incentives in a quest based learning environment. This environment is purely for engaging students with activities and consequently STEMS subjects. The game-based approach encourages the users to perform more and more activities. It also provides scaffolding and support in regards to undertaking the quests and building the rigs. The RALfie system thus ensures collaboration both actively e.g. building the rigs together and passively by using others creations.

Each quest is represented as a game that poses a challenge to the user. The user must navigate through the series of steps on the UI of the experiment to complete the challenges which completes the earing outcome. Once an 
experiment is completed, the user can move on the next in the next one.

\section{THE OPEN APPROACH}

The P2P Architecture allows for an open-ended system with no predetermined limitations of what experiments can be added to the system..

\section{A. Challenges for Experiment Hosting}

Apart from building the rigs, hosting the experiments poses additional challenges for makers. They have to

1. learn to create and maintain the experiment at least for some duration of time. This gives the makers scope to learn about reliability of the setup and to investigate possibilities to improve on the rig design.

2. create an experiment that is usable by others. This means that the interface has to be made in such a way that it is correctly interpreted by others. The also offers opportunities to explore different pedagogical approaches when designing the user interface.

The open system allows for a variety of experiments to be built. This also means that the same experiments can be built in many ways as the makers see fit. This allows the makers to create things that may not otherwise be directly part of the curriculum. All of these features allow for makers to have an enriched experience. For users that are not building experiments themselves, it can be of value to look at different examples of the same experiment.

\section{B. Limitations}

The main limimtation of this approach is the quality and availably of experiments. As the experiments are built by layman reliability can be an issue. While this can be frustrating for users, it provides great leaning opportunities for makers. The second concern is that experiments are not expected to be available for extended periods; although many users will be able to make the same rig, thus increasing their availability.

\section{CONCLUSIONS}

RALfie is a peer-to-peer remote access laboratory system where students are both users of experiments as well as makers. The system allows direct connections between learner and makers' experiential rigs. A graphical programming language is the used to build the user interface and control logic. Interaction with the environment is supported by a quest-based learning environment, presenting experiments as a set of hierarchical groups of activi- ties. This hands-on experience to build any experimental setup and provides opportunities to collaborate with fellow students. While RALfie is normally used by children, the approach is also applicable to older students.

\section{REFERENCES}

[1] A. Maiti, A. D. Maxwell, and A. A. Kist, "Features, Trends and Characteristics of Remote Access Laboratory Management Systems," International Journal of Online Engineering, vol. 10, pp. 31-37, 2014. http://dx.doi.org/10.3991/ijoe.v10i2.3221

[2] D. Lowe, P. Newcombe, and B. Stumpers, "Evaluation of the Use of Remote Laboratories for Secondary School Science Education," Research in Science Education, vol. 43, pp. 1197-1219, 2013/06/01 2013.

[3] A. A. Kist, A. Maiti, A. D. Maxwell, L. Orwin, W. Midgley, K. Noble, et al., "Overlay network architectures for peer-to-peer Remote Access Laboratories," in Remote Engineering and Virtual Instrumentation (REV), 2014 11th International Conference on, 2014, pp. 274-280. http://dx.doi.org/10.1109/rev.2014.6784274

[4] A. Maiti, A. D. Maxwell, A. A. Kist, and L. Orwin, "Integrating enquiry-based learning pedagogies and remote access laboratory for STEM education," in Global Engineering Education Conference (EDUCON), 2014 IEEE, 2014, pp. 706-712. http://dx.doi.org/10.1109/educon.2014.6826171

[5] A. Maiti, A. A. Kist, and A. D. Maxwell, "Using Network Enabled Microcontrollers in Experiments for a Distributed Remote Laboratory," presented at the REV 2014, Porto, Portugal, 2014. http://dx.doi.org/10.1109/rev.2014.6784250

[6] K. Brennan, "Scratch-Ed: an online community for scratch educators," presented at the Proceedings of the 9th international conference on Computer supported collaborative learning - Volume 2, Rhodes, Greece, 2009. http://dx.doi.org/10.3115/1599503. 1599529

\section{AUTHORS}

A. Kist, A. Maiti, A. Maxwell and R. Burtenshaw are with the School of Mechanical and Electrical Engineering, Faculty of HES, University of Southern Queensland, Australia (andrew.maxwell@usq.edu.au, anandamaiti@live.com, kist@ieee.org)

Lidy Orwin is with the ADFI, University of Southern Queensland, Australia (lindy.orwin@usq.edu.au)

W. Ting and P. Albion are with the School of Teacher Education and Early Childhood, University of Southern Queensland, Australia (wu.ting@usq.edu.au, peter.albion@usq.edu.au )

This work is supported through the Australian governments Collaborative Research Network - Digital Futures Program.

Submitted, 28 September 2015. Published as resubmitted by the authors on 12 January 2016. 2015. 